\title{
Analysis of lipoprotein profiles of healthy cats by gel-permeation high-performance liquid chromatography
}

\author{
Hisashi MIZUTANI ${ }^{1) *}$, Toshinori SAKO ${ }^{2)}$, Hiroko OKUDA ${ }^{3)}$, Nobuaki ARAI ${ }^{4)}$, Koji KURIYAMA ${ }^{5)}$, Akihiro MORI $^{2)}$, \\ Itaru YOSHIMURA ${ }^{6)}$ and Hidekazu KOYAMA ${ }^{1)}$ \\ 1) Veterinary Internal Medicine, Nippon Veterinary and Life Science University, 1-7-1 Kyonan, Musashino, Tokyo 180-8602, Japan \\ ${ }^{2)}$ Department of Veterinary Nursing, Nippon Veterinary and Life Science University, 1-7-1 Kyonan, Musashino, Tokyo 180-8602, Japan \\ 3) Okuda Animal Hospital, 5-28-6 Kamoi, Midori-ku, Yokohama City, Kanagawa 226-0003, Japan \\ 4) Spectrum Lab, Japan K.K., 1-5-22-201 Midoriga-Oka, Meguro-ku, Tokyo 152-0034, Japan \\ ${ }^{5)}$ Skylight Biotech K.K., 100-4 Iijima-Sunada, Akita-city, Akita 011-0911, Japan \\ ${ }^{6)}$ Fuji Animal Farm, Nippon Veterinary and Life Science University, 799 Fujigane, Fuji-Kawaguchiko-cho, Yamanashi 401-0338, Japan
}

(Received 28 January 2016/Accepted 20 April 2016/Published online in J-STAGE 7 May 2016)

ABSTRACT. Density gradient ultracentrifugation (DGUC) and gel electrophoresis are conventionally used to obtain lipoprotein profiles of animals. We recently applied high-performance liquid chromatography with a gel permeation column (GP-HPLC) and an on-line dual enzymatic system to dogs for lipoprotein profile analysis. We compared the GP-HPLC with DGUC as a method to obtain a feline lipoprotein profile. The lipoprotein profiles showed large and small peaks, which corresponded to high-density lipoprotein (HDL) and low-density lipoprotein (LDL), respectively, whereas very low-density lipoprotein (VLDL) and chylomicron (CM) were only marginally detected. This profile was very similar to that of dogs reported previously. Healthy cats also had a small amount of cholesterol-rich particles distinct from the normal LDL or HDL profile. There was no difference in lipoprotein profiles between the sexes, but males had a significantly larger LDL particle size $(P=0.015)$. This study shows the feasibility of GP-HPLC for obtaining accurate lipoprotein profiles with small sample volumes and provides valuable reference data for healthy cats that should facilitate diagnoses.

KEY WORDS: cat, HPLC, lipoprotein profile, triglyceride

doi: 10.1292/jvms.16-0052; J. Vet. Med. Sci. 78(8): 1277-1281, 2016

Lipoproteins are classified based on their density, particle size and electrophoretic mobility [21]. In human medicine, many detection methods have been developed for lipoprotein profiling. Conventionally, density gradient ultracentrifugation (DGUC) has been the most widely used procedure for lipoprotein fractionation [11], but this method is difficult to apply in clinical tests because it is a time-consuming and complicated procedure requiring a large sample volume [21]. Polyacrylamide or agarose gel electrophoresis-based methods have also been described and clinically applied for human applications $[18,21]$. However, because animal lipoproteins are not well characterized and their compositions are different from those of humans, it is often difficult to separate animal lipoproteins using conventional electrophoresis procedures developed for humans. We previously reported the use of a simple and high-quality method for detecting a canine lipoprotein profile using a high-performance liquid chromatography system with gel permeation columns (GP-HPLC) and on-line dual enzymatic detection, which was originally described by Usui et al. [21]. The aim of the present study was to confirm the feasibility of this

*Correspondence to: Mizutani, H., Veterinary Internal Medicine, Nippon Veterinary and Life Science University, 1-7-1 Kyonan, Musashino, Tokyo 180-8602, Japan. e-mail: hisashi-m@nvlu.ac.jp (C) The Japanese Society of Veterinary Science

This is an open-access article distributed under the terms of the Creative Commons Attribution Non-Commercial No Derivatives (by-nc-nd) License $<$ http://creativecommons.org/licenses/by-nc-nd/4.0/>. method for obtaining the lipoprotein profile of cats. These data should also help to provide a standardized lipoprotein profile of healthy cats.

\section{MATERIALS AND METHODS}

To compare the lipoprotein profiles obtained using the DGUC and GP-HPLC methods, blood samples were obtained from three healthy Japanese domestic cats (4 years old, male and body weight approximately $4 \mathrm{~kg}$ ) that were maintained in our laboratory at the Nippon Veterinary and Animal Science University. Blood samples for determination of standard lipoprotein profiles of cats were obtained from 20 healthy Japanese domestic cats (11 males; $5.9 \pm$ 4.2 years and 9 females: $5.5 \pm 4.5$ years) that were brought to the Okuda Animal Hospital for standard health examinations. Clinical observations and general blood examination (complete blood counts and biochemical analysis conducted by the Yokohama Laboratory of Lance Ltd. (Yokohama, Japan) revealed no abnormalities in any of the cats. Blood samples were collected from the jugular vein after fasting for more than $10 \mathrm{hr}$ and before feeding in the morning. Plasma samples treated with EDTA were isolated by centrifugation at $1,800 \times g$ for $10 \mathrm{~min}$. DGUC was performed immediately after plasma separation, whereas other analyses were carried out within 3 days of sample collection and the samples were stored at $4^{\circ} \mathrm{C}$.

The dual-detection GP-HPLC method for lipoprotein profile analysis was described previously $[16,18,21]$. This 
analysis was performed by Skylite Biotech Corporation in their laboratory (Akita, Japan). The DGUC method and agarose gel electrophoresis were performed as previously described for dogs [16] using commercial kits (Titan III gel system with an Fat Red 7B staining kit; Helena Laboratories; Saitama, Japan and Calibrated Densitometer GS-800; BioRad Laboratories; Hercules, CA, U.S.A.).

Statistical analysis for comparison between sexes was carried out by the Student's $t$-test using the StatView program (version 5.0, Japanese Edition; SAS Institute Inc., Cary, NC, U.S.A.). Data are presented as mean $\pm \mathrm{SD}$.

\section{RESULTS}

The lipid profiles of the whole plasma of Cat 1 as determined by GP-HPLC and DGUC are shown in Fig. 1A and 1-B, respectively. As shown in Figure, the cholesterol curves obtained from both analytical methods provided two peaks, one large peak and one small peak. Fraction \#8 of Fig. 1B was analyzed by GP-HPLC, and a single peak was eluted at 28-29 min (Fig. 1C). Fraction \#14 showed a small peak eluted at around $24 \mathrm{~min}$ and smaller peak at around $28 \mathrm{~min}$ by GP-HPLC (Fig. 1D). The result of agarose gel electrophoresis from the Cat1 sample is shown in Fig. 1E, which presents the electrophoretic patterns of the whole plasma and the DGUC fractions (\#2, 5, 8, 12, 14, 20, 26 and 27). Fractions $\# 2,5$ and 8 , which correspond to the larger peak, produced a single band at the position corresponding to $\alpha$-lipoprotein (HDL), and Fractions \#12 and 14, which produced a smaller peak, showed two bands at the $\alpha$-and $\beta$-lipoprotein (LDL) positions. However, any band was not obtained from fraction \#20. Fractions \#26 and 27 produced a broad band from the application point (chylomicron [CM]) to the pre- $\beta$ (very lowdensity lipoprotein [VLDL]) position. Therefore, the larger fraction that peaked at around 28-29 $\mathrm{min}$ by GC-HPLC $=$ was identified as HDL. The smaller peak at 24-25 min was also observed in this investigation. As shown in Table 1, the concentrations of lipids in whole plasma as determined by DGUC and GP-HPLC were compared with those in each lipoprotein fraction analyzed (Table 1). As shown in Table, the little difference was observed in cholesterol levels estimated with the DGCU and GP-HPLC method. In contrast, larger differences were observed with respect to the TG level of each fraction. The larger difference was especially true in the VLDL+CM fraction.

The lipid concentrations in each lipoprotein and the particle sizes determined by GP-HPLC of the 20 healthy cats are shown in Tables 2 and 3, respectively. There was no difference in lipoprotein profiles between males and females, but males had a significantly larger LDL particle size $(P=0.015)$.

\section{DISCUSSION}

The cholesterol curves obtained from both analytical methods, GP-HPLC and DGUC, provided two peaks, one large peak and one small peak, which was a similar pattern to that observed in dogs in our previous study [16]. Fraction \#8 with a larger peak corresponding to the high-density lipoprotein (HDL) fraction according to previous reports $[1,15,22]$, was analyzed by GP-HPLC, and a single peak was eluted at 28-29 min. Fraction \#14, which comprised the low-density lipoprotein (LDL) fraction, showed a small peak eluted at around $24 \mathrm{~min}$ and smaller peak at around $28 \mathrm{~min}$ by GP-HPLC. Usui et al. [21] also reported the outflow of endogenous free glycerol after HDL elution. The peak observed at around $36 \mathrm{~min}$ in GP-HPLC whole plasma analysis was considered to be free glycerin.

We considered the smaller peak at 24-25 min to be LDL. However, when the LDL fraction separated by DGUC was analyzed using the GP-HPLC method, it was partially included in the small and cholesterol-rich lipoprotein component. Agarose gel electrophoresis revealed 2 bands for this LDL fraction, at the $\beta$ and $\alpha$ positions. These observations indicated that it is impossible to accurately separate the cat LDL by DGUC. The small particles, which showed the specific gravity of LDL, were considered to be apolipoprotein E-rich HDL (ApoE-rich HDL) or small, dense LDL (sdLDL). The gravity of sdLDL is the same as that of normal LDL, but the particle size is smaller; this lipoprotein fraction is closely related to metabolic syndrome in humans $[6-7,17$, 20]. On the other hand, ApoE-rich HDL, which consists of cholesterol-rich particles with a low-gravity HDL and is observed in cholesterol ester transfer protein (CETP)-deficient patients [3, 23], was electrophoresed into $\alpha$-lipoprotein on the agarose gel. Because cats have low CETP activity [10], this result suggests that ApoE-rich HDL is also observed in healthy cats. In this study, we could not determine whether this fraction was sdLDL or ApoE-rich HDL; nonetheless, the results suggest that healthy cats have a small amount of cholesterol-rich particles that are distinct from normal LDL or HDL.

Because our DGUC method could not accurately separate CM and VLDL in cats, it was not possible to confirm their elution times by the GP-HPLC method using DGUC-separated fraction samples. However, the gradual triglyceride (TG) peak observed near the 22-min mark in whole plasma analyzed with GP-HPLC method was considered to be derived from VLDL particles based on particle size. In addition, the TG peak produced at around $18 \mathrm{~min}$ was considered to correspond to CM.

Little difference was observed in cholesterol and TG levels estimated with the DGUC and GP-HPLC methods. Using the DGUC method, the TG-rich lipoprotein fraction was previously reported to adhere to the wall of the centrifugation tube, which could lead to quantification errors [21]. The large variability in the VLDL+CM fraction between DGUC and GP-HPLC was likely caused by the large particle size of the cat's CM, which is greater than the maximum particle size that can pass through the GP-HPLC column. Therefore, it would be useful to confirm whether there is any deviation between the values obtained for total plasma TG levels as measured by GP-HPLC and an enzymatic assay method for general blood biochemistry to accurately evaluate the TGrich lipoprotein profile with the GP-HPLC method.

There was no difference between lipoprotein profiles of males and that of females, but lipoprotein profiles of males 
A

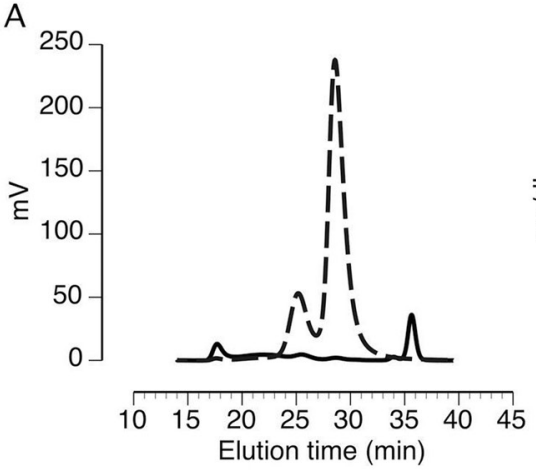

B

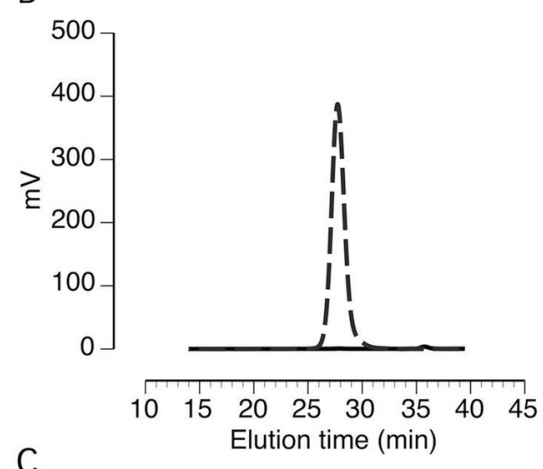

C

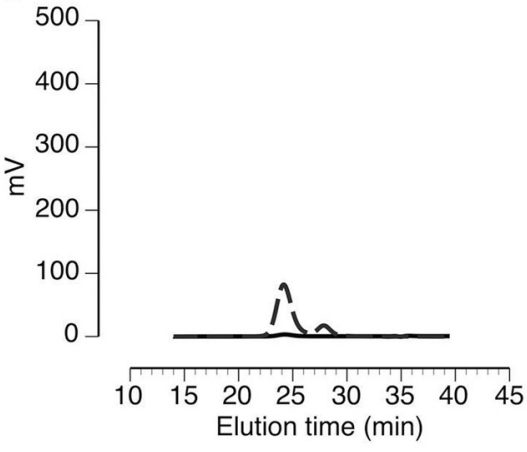

D
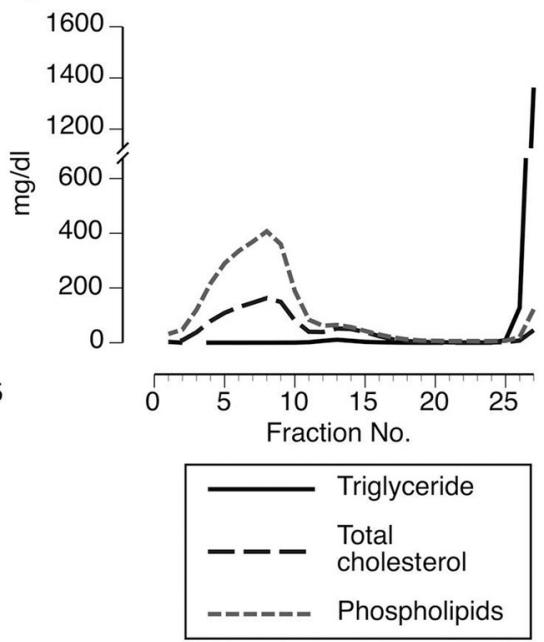

$\mathrm{E}$

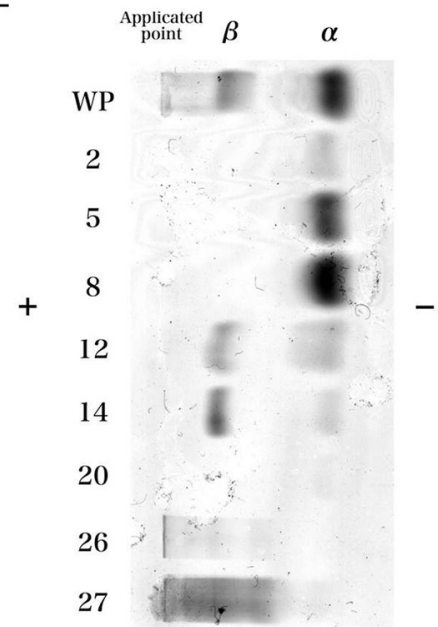

Fig. 1. The results of lipoprotein analyses in Cat 1 (4-year-old male, healthy Japanese domestic cat). The lipoprotein profiles of whole plasma acquired by (A) high-performance liquid chromatography with gel permeation columns (GP-HPLC) and (B) density gradient ultracentrifugation (DGUC). (C and D) GP-HPLC pattern of the high-density lipoprotein (HDL) fraction (Fraction \#8) and the low-density lipoprotein (LDL) fraction (Fraction \#14) separated by the DGUC method. (E) Agarose gel electrophoretic patterns of whole plasma (WP) and several fractions ( $\# 2,5,8,12,14,20,26$ and 27).

had a significantly larger LDL particle size $(P=0.015)$. Gender differences of LDL particle size have been reported in human $[2,19]$. It was reported that serum triglyceride concentration $[2,4,13,19]$, insulin resistance $[4,13]$ and proinsulin concentration [4] affect average LDL particle size. While gender differences of these factors are considered to lead to gender differences of LDL particle size. In the present study, we did not verify these factors. This problem will be future challenges. The results indicate that the healthy cats' lipoprotein profile could be characterized as an HDLrich profile, i.e., high HDL, low LDL, and marginal VLDL and CM contents were detected. This pattern is similar to that of healthy dogs reported previously [16], although the cat's LDL had a lower TG content compared to that of dogs.

Development of hyperlipidemia is often related to endocrine disease. Human type 2 diabetes mellitus patients often present hyperlipidemia $[8,14]$, and we previously reported that diabetic dogs had high concentrations of VLDL-TG and VLDL-cholesterol [16]. Similar to dogs and humans, many of the lipid metabolism abnormalities observed in cats are caused by endocrine diseases, such as diabetes [5]. Furthermore, congenital anomalies with hyperlipidemia, such 
Table 1. Concentrations of lipids in each lipoprotein fraction as analyzed by DGUC and GPHPLC in healthy cats

\begin{tabular}{|c|c|c|c|c|c|c|c|c|}
\hline & \multirow{2}{*}{ Item } & \multirow{2}{*}{ Unit } & \multicolumn{3}{|c|}{ GP-HPLC } & \multicolumn{3}{|c|}{ DGUC } \\
\hline & & & Cat 1 & Cat 2 & Cat 3 & Cat 1 & Cat 2 & Cat 3 \\
\hline \multirow[t]{3}{*}{ HDL } & T-cho & $\mathrm{mg} / \mathrm{d} l$ & 138.1 & 119.8 & 95.3 & 117.5 & 123.8 & 96.7 \\
\hline & TG & $\mathrm{mg} / \mathrm{d} l$ & 4.7 & 3.5 & 2.8 & 0.5 & 1.2 & 1.4 \\
\hline & PL & $\mathrm{mg} / \mathrm{d} l$ & 344.0 & 308.5 & 245.6 & 303.9 & 312.4 & 243.6 \\
\hline \multirow[t]{3}{*}{ LDL } & T-cho & $\mathrm{mg} / \mathrm{d} l$ & 34.8 & 24.0 & 16.7 & 30.3 & 14.0 & 11.9 \\
\hline & TG & $\mathrm{mg} / \mathrm{d} l$ & 10.2 & 8.9 & 12.3 & 3.9 & 3.0 & 6.0 \\
\hline & PL & $\mathrm{mg} / \mathrm{d} l$ & 41.4 & 30.9 & 25.8 & 41.1 & 17.8 & 22.4 \\
\hline \multirow[t]{3}{*}{ VLDL } & T-cho & $\mathrm{mg} / \mathrm{d} l$ & 3.8 & 3.5 & 3.0 & & & \\
\hline & TG & $\mathrm{mg} / \mathrm{d} l$ & 21.1 & 14.3 & 27.0 & & & \\
\hline & PL & $\mathrm{mg} / \mathrm{d} l$ & 5.3 & 5.7 & 5.6 & & & \\
\hline \multirow[t]{3}{*}{$\mathrm{CM}$} & T-cho & $\mathrm{mg} / \mathrm{d} l$ & 0.6 & 0.1 & 0.2 & & & \\
\hline & TG & $\mathrm{mg} / \mathrm{d} l$ & 14.0 & 2.2 & 6.0 & & & \\
\hline & PL & $\mathrm{mg} / \mathrm{d} l$ & 1.1 & 0.2 & 0.0 & & & \\
\hline \multirow[t]{3}{*}{$\mathrm{VLDL}+\mathrm{CM}$} & T-cho & $\mathrm{mg} / \mathrm{d} l$ & & & & 6.3 & 5.1 & 3.0 \\
\hline & $\mathrm{TG}$ & $\mathrm{mg} / \mathrm{d} l$ & & & & 114.7 & 28.9 & 65.4 \\
\hline & PL & $\mathrm{mg} / \mathrm{d} l$ & & & & 18.7 & 10.7 & 15.9 \\
\hline \multirow[t]{3}{*}{ Total } & T-cho & $\mathrm{mg} / \mathrm{d} l$ & 177.4 & 147.5 & 115.2 & 169.1 & 142.9 & 112.6 \\
\hline & TGIF & $\mathrm{mg} / \mathrm{d} l$ & 50.0 & 28.9 & 49.1 & 119.6 & 33.2 & 72.7 \\
\hline & PL & $\mathrm{mg} / \mathrm{d} l$ & 391.7 & 345.3 & 277.0 & 341.0 & 341.0 & 281.9 \\
\hline
\end{tabular}

HDL: high-density lipoprotein; LDL: low-density lipoprotein; VLDL: very low-density lipoprotein; CM: chylomicron. T-cho: total cholesterol; TG: triglyceride; PL: phospholipids. GP-HPLC: highperformance liquid chromatography with gel permeation columns. DGUC: density gradient ultracentrifugation.

Table 2. Comparison of lipoprotein profiles between male $(n=9)$ and female $(n=11)$ healthy cats. Data are mean \pm SD. $P$-values were calculated by the Student's $t$-test (male vs. female)

\begin{tabular}{lllcccc}
\hline Lipoprotein & Item & Unit & Males $(\mathrm{n}=9)$ & Females $(\mathrm{n}=11)$ & $P$-value & Total $(\mathrm{n}=20)^{*}$ \\
\hline HDL & T-cho & $\mathrm{mg} / \mathrm{d} l$ & $130.4 \pm 47.6$ & $125.1 \pm 19.7$ & 0.737 & $128 \pm 35$ \\
& TG & $\mathrm{mg} / \mathrm{d} l$ & $4.6 \pm 3.9$ & $4.4 \pm 1.9$ & 0.88 & $4.6 \pm 2.9$ \\
LDL & T-cho & $\mathrm{mg} / \mathrm{d} l$ & $26.9 \pm 25.1$ & $17.4 \pm 8.3$ & 0.251 & $22.54 \pm 18.1$ \\
& TG & $\mathrm{mg} / \mathrm{d} l$ & $13.3 \pm 5.9$ & $13.5 \pm 4.7$ & 0.927 & $13.4 \pm 5$ \\
VLDL & T-cho & $\mathrm{mg} / \mathrm{d} l$ & $2.8 \pm 1.6$ & $2.2 \pm 1.2$ & 0.347 & $2.4 \pm 1.4$ \\
& TG & $\mathrm{mg} / \mathrm{d} l$ & $29.8 \pm 26$ & $19.3 \pm 12.6$ & 0.251 & $23.4 \pm 19.6$ \\
CM & T-cho & $\mathrm{mg} / \mathrm{d} l$ & $0.1 \pm 0.1$ & $0.1 \pm 0.3$ & 0.502 & $0.1 \pm 0.2$ \\
\multirow{2}{*}{ Total } & TG & $\mathrm{mg} / \mathrm{d} l$ & $5.7 \pm 11.6$ & $2.7 \pm 3.6$ & 0.417 & $3.9 \pm 7.9$ \\
& T-cho & $\mathrm{mg} / \mathrm{d} l$ & $160.3 \pm 68.2$ & $144.7 \pm 26.3$ & 0.494 & $153.2 \pm 49.7$ \\
& TG & $\mathrm{mg} / \mathrm{d} l$ & $53.3 \pm 44.3$ & $39.8 \pm 17.4$ & 0.364 & $45.6 \pm 33$ \\
\hline
\end{tabular}

HDL: high-density lipoprotein; LDL: low-density lipoprotein; VLDL: very low-density lipoprotein;CM: chylomicron; T-cho: total cholesterol; TG: triglyceride.

Table 3. Comparison of lipoprotein particle sizes between male and female healthy cats. Data are mean $\pm \mathrm{SD}$. $P$-values were calculated by the Student's $t$-test (male vs. female)

\begin{tabular}{lccccc}
\hline \multicolumn{2}{c}{ Lipoprotein Unit } & Male $(\mathrm{n})$ & Female $(\mathrm{n})$ & $P$-value & Total $(\mathrm{n})$ \\
\hline HDL & $\mathrm{nm}$ & $11.4 \pm 0.5(9)$ & $11.1 \pm 0.3(11)$ & 0.164 & $11.2 \pm 0.4(20)$ \\
LDL & $\mathrm{nm}$ & $22.8 \pm 0.6(8)$ & $21.8 \pm 0.8(10)$ & 0.015 & $22.3 \pm 0.9(19)$ \\
VLDL & $\mathrm{nm}$ & $40.2 \pm 1.4(8)$ & $40 \pm 2.5(11)$ & 0.789 & $2.2(19)$ \\
\hline
\end{tabular}

HDL: high-density lipoprotein; LDL: low-density lipoprotein; VLDL: very lowdensity lipoprotein. 
as LPL deficiency [9, 12], have also been reported in cats. Collectively, our results show that the GP-HPLC method can produce accurate lipoprotein profiles using a small sample volume and thus shows good potential for research and diagnosis of dyslipidemia in cats.

CONFLICT OF INTEREST. This study was conducted by the fund of Skylight Biotech K.K. There is not any conflict of interest related to this research.

ACKNOWLEDGMENT. Authors thank Dr. Mariko Mochizuki-Kobayashi (Department of Veterinary Nursing, Nippon Veterinary and Life Science University) for supports of construction of this report.

\section{REFERENCES}

1. Brocks, D. R., Ramaswamy, M., MacInnes, A. I. and Wasan, K. M. 2000. The stereoselective distribution of halofantrine enantiomers within human, dog, and rat plasma lipoproteins. Pharm. Res. 17: 427-431. [Medline] [CrossRef]

2. Campos, H., Blijlevens, E., McNamara, J. R., Ordovas, J. M., Posner, B. M., Wilson, P. W., Castelli, W. P. and Schaefer, E. J. 1992. LDL particle size distribution. Results from the Framingham Offspring Study. Arterioscler. Thromb. 12: 1410-1419. [Medline] [CrossRef]

3. Chiba, H., Eto, M., Fujisawa, S., Akizawa, K., Intoh, S., Miyata, O., Noda, K., Matsuno, K. and Kobayashi, K. 1993. Increased plasma apolipoprotein E-rich high-density lipoprotein and its effect on serum high-density lipoprotein cholesterol determination in patients with familial hyperalphalipoproteinemia due to cholesteryl ester transfer activity deficiency. Biochem. Med. Metab. Biol. 49: 79-89. [Medline] [CrossRef]

4. Festa, A., D’Agostino, R. Jr., Mykkänen, L., Tracy, R. P., Hales, C. N., Howard, B. V. and Haffner, S. M. 1999. LDL particle size in relation to insulin, proinsulin, and insulin sensitivity. The Insulin Resistance Atherosclerosis Study. Diabetes Care 22: 1688-1693. [Medline] [CrossRef]

5. Ford, R. B. and Ludlow, C. L. 2010. Disorders of lipid metabolism. pp. 545-557. In: Small Animal Clinical Nutrition (Hand, M. S., Thatcher, C. D., Remillard, R. L., Roudebush, P. and Novotny, B. J. eds.), Mark Morris Institute, Topeka.

6. Gazi, I., Tsimihodimos, V., Filippatos, T., Bairaktari, E., Tselepis, A. D. and Elisaf, M. 2006. Concentration and relative distribution of low-density lipoprotein subfractions in patients with metabolic syndrome defined according to the National Cholesterol Education Program criteria. Metabolism 55: 885-891. [Medline] [CrossRef]

7. Gazi, I., Tsimihodimos, V., Filippatos, T. D., Saougos, V. G., Bairaktari, E. T., Tselepis, A. D. and Elisaf, M. 2006. LDL cholesterol estimation in patients with the metabolic syndrome. Lipids Health Dis. 5: 8. [Medline] [CrossRef]

8. Ginsberg, H. N. 1991. Lipoprotein physiology in nondiabetic and diabetic states. Relationship to atherogenesis. Diabetes Care 14: 839-855. [Medline] [CrossRef]

9. Ginzinger, D. G., Lewis, M. E., Ma, Y., Jones, B. R., Liu, G. and Jones, S. D. 1996. A mutation in the lipoprotein lipase gene is the molecular basis of chylomicronemia in a colony of domestic cats. J. Clin. Invest. 97: 1257-1266. [Medline] [CrossRef]
10. Guyard-Dangremont, V., Desrumaux, C., Gambert, P., Lallemant, C. and Lagrost, L. 1998. Phospholipid and cholesteryl ester transfer activities in plasma from 14 vertebrate species. Relation to atherogenesis susceptibility. Comp. Biochem. Physiol. B Biochem. Mol. Biol. 120: 517-525. [Medline] [CrossRef]

11. Havel, R. J., Eder, H. A. and Bragdon, J. H. 1955. The distribution and chemical composition of ultracentrifugally separated lipoproteins in human serum. J. Clin. Invest. 34: 1345-1353. [Medline] [CrossRef]

12. Jones, B. R., Wallace, A., Harding, D. R., Hancock, W. S. and Campbell, C. H. 1983. Occurrence of idiopathic, familial hyperchylomicronaemia in a cat. Vet. Rec. 112: 543-547. [Medline] [CrossRef]

13. Kobayashi, H., Nakamura, T., Miyaoka, K., Nishida, M., Funahashi, T., Yamashita, S. and Matsuzawa, Y. 2001. Visceral fat accumulation contributes to insulin resistance, small-sized low-density lipoprotein, and progression of coronary artery disease in middle-aged non-obese Japanese men. Jpn. Circ. J. 65: 193-199. [Medline] [CrossRef]

14. Kreisberg, R. A. 1998. Diabetic dyslipidemia. Am. J. Cardiol. 82: $67 \mathrm{U}-73 \mathrm{U}$; discussion $85 \mathrm{U}-86 \mathrm{U}$.

15. Lehmann, R., Bhargava, A. S. and Günzel, P. 1993. Serum lipoprotein pattern in rats, dogs and monkeys, including method comparison and influence of menstrual cycle in monkeys. Eur. J. Clin. Chem. Clin. Biochem. 31: 633-637. [Medline]

16. Mizutani, H., Sako, T., Arai, N., Kuriyama, K., Yoshimura, I., Mori, A., Iwase, K. and Hirose, H. 2010. Application of gel permeation HPLC for lipoprotein profiling in dogs. J. Vet. Med. Sci. 72: 813-817. [Medline] [CrossRef]

17. Nikolic, D., Katsiki, N., Montalto, G., Isenovic, E. R., Mikhailidis, D. P. and Rizzo, M. 2013. Lipoprotein subfractions in metabolic syndrome and obesity: clinical significance and therapeutic approaches. Nutrients 5: 928-948. [Medline] [CrossRef]

18. Okazaki, M., Usui, S., Ishigami, M., Sakai, N., Nakamura, T., Matsuzawa, Y. and Yamashita, S. 2005. Identification of unique lipoprotein subclasses for visceral obesity by component analysis of cholesterol profile in high-performance liquid chromatography. Arterioscler. Thromb. Vasc. Biol. 25: 578-584. [Medline] [CrossRef]

19. Rainwater, D. L., Mitchell, B. D., Comuzzie, A. G. and Haffner, S. M. 1999. Relationship of low-density lipoprotein particle size and measures of adiposity. Int. J. Obes. Relat. Metab. Disord. 23: 180-189. [Medline] [CrossRef]

20. Rizzo, M. and Berneis, K. 2007. Small, dense low-densitylipoproteins and the metabolic syndrome. Diabetes Metab. Res. Rev. 23: 14-20. [Medline] [CrossRef]

21. Usui, S., Hara, Y., Hosaki, S. and Okazaki, M. 2002. A new online dual enzymatic method for simultaneous quantification of cholesterol and triglycerides in lipoproteins by HPLC. J. Lipid Res. 43: 805-814. [Medline]

22. Wasan, K. M., Ramaswamy, M., Ng, S. P., Wong, W., Parrott, S. C., Ojwang, J. O., Wallace, T. and Cossum, P. A. 1998. Differences in the lipoprotein distribution of free and liposomeassociated all-trans-retinoic acid in human, dog, and rat plasma are due to variations in lipoprotein lipid and protein content. Antimicrob. Agents Chemother. 42: 1646-1653. [Medline]

23. Yamashita, S., Sprecher, D. L., Sakai, N., Matsuzawa, Y., Tarui, S. and Hui, D. Y. 1990. Accumulation of apolipoprotein E-rich high density lipoproteins in hyperalphalipoproteinemic human subjects with plasma cholesteryl ester transfer protein deficiency. J. Clin. Invest. 86: 688-695. [Medline] [CrossRef] 